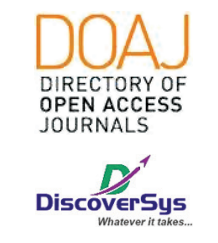

Published by DiscoverSys

\title{
Acute thoracic aortic dissection: A case report
}

\author{
Dewa Ayu Ratna Dewi, ${ }^{1 *}$ Made Dwija Putra Ayusta²
}

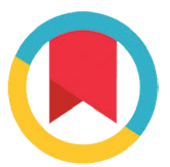

CrossMark
ABSTRACT

Introduction: Aortic dissection (AD) is the most common aortic emergency, result in a catastrophic outcome. Patients usually asymptomatic, some of them present with sudden severe, tearing or sharp back or anterior chest pain and haemodynamic disturbance. Early and accurate diagnosis and treatment determine the patient outcome.

Case Report: A case of 40 years old man complained of acute, sharp, chest pain since 4 days before admission which worsen instead of medical treatment, with a history of smoking since a few years ago. A vital sign was within normal limit. Laboratory result showed high CKMB, troponin T, LDH result. Occasional ventricular extrasystoles and inferior ischaemic was found in the ECG examination. From echocardiography, the patient suspected to have aortic dissection Stanford type A since an intimal flap on ascending aorta was found, with left ventricle hypertrophy, dilatation of right atrium and right ventricle, severe aortic regurgitation, moderate mitral regurgitation, severe tricuspid regurgitation, moderate pulmonary regurgitation and moderate pulmonary hypertension. From plain radiography, the patient showed mediastinum widening and cardiomegaly, CT angiography showed Stanford type A aortic dissection (DeBakey type I). The patient refused to seek further treatment for operation as suggested.

Conclusion: It is still challenging to differentiate aortic dissection (AD) and another acute chest emergency. Since clinical presentation and laboratory result may be similar between those illnesses. Therefore careful history taking and further imaging examination should be considered to increase the patient outcome.

Keywords: chest pain, aortic dissection, Stanford classification, De Bakey classification.

Cite This Article: Dewi, D.A.R., Ayusta, M.D.P. 2020. Acute thoracic aortic dissection: A case report. Intisari Sains Medis 11(2): 769-772. D0l: 10.15562/ism.v11i2.715

\section{INTRODUCTION}

Aortic dissection (AD) is one of the most common acute non-traumatic emergency of aortic with high mortality, which increases in patients with complicated dissection. The aetiology is frequently unknown but is related to hypertension $(72 \%)$ and atherosclerosis (30\%). ${ }^{1,2}$ Other predisposing factors are Marfan's syndrome, Ehlers-Danlos syndrome, coarctation, bicuspid aortic valve, Turner syndrome, aortitis, cocaine abuse, pregnancy and blunt chest trauma. ${ }^{2}$ The prevalence of AD has been reported approximately 5200/100.000 in Western countries, with a male predominance of 3:1. And in approximately two-thirds of a case involving the ascending aorta. ${ }^{3}$

$\mathrm{AD}$ is the result of a sudden longitudinal detachment of the aortic intima and adventitia causing infiltration of blood to the medial layer, which becomes the false lumen and resulting two lumina in the blood vessel: true and false lumen., ${ }^{1,2} \mathrm{AD}$ is classified to type A and type B which depends on the ascending aorta involvement. ${ }^{2,4}$ Dissection type and extension and occurrence of associated complications determined the outcome. ${ }^{2}$

The symptoms of $\mathrm{AD}$ may be variable and can mimic other more common conditions such as myocardial ischemia. ${ }^{5}$ Around $10 \%$ of acute AD is asymptomatic, some complained of only mild pain and others may come with acute onset of severe chest pain that is defined as sharp and tearing. All patient presenting with such chest pain should be excluded from the possibility of thoracic AD. Thus it is very important confirming the diagnosis with the appropriate imaging studies, as misdiagnosis may lead to fatal outcomes. ${ }^{5}$

\section{CASE REPORT}

A 40-years-old man referred from district hospital where acute myocardial infarction was diagnosed. He suddenly developed acute chest pain since 4 days before admission. The pain was said to be an acute sharp chest pain that radiates, to the neck and left arm and associated with nausea, sweating, and diaphoresis. The pain worsens instead of medical treatment. The patient had a history of smoking approximately 20 cigarettes per day since years ago, without any known systemic disease in the past. Initial examination showed blood pressure of $90 / 60 \mathrm{mmHg}$, heart rate of 70 beats per minute, respiratory rate was 20 breath per minute, the temperature of $36^{\circ} \mathrm{C}$ and oxygen saturation of $96 \%$ breathing ambient air. No abnormalities were found on examination of the heart, lungs, or abdomen. Patient showed an asymmetrical bilateral peripheral pulse. 


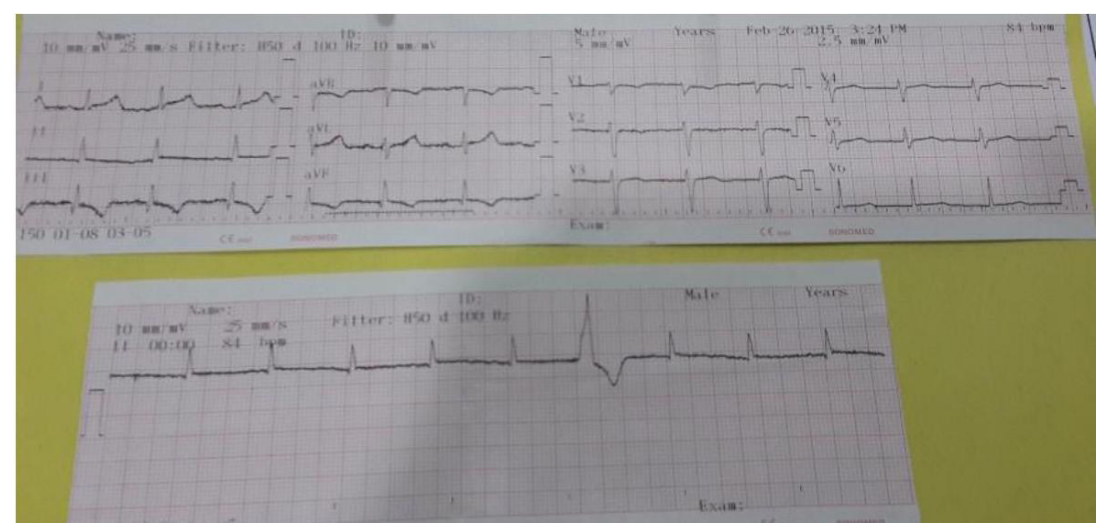

Figure 1 Electrocardiogram showing T inversion in III-AVF and occasional ventricular extrasystole
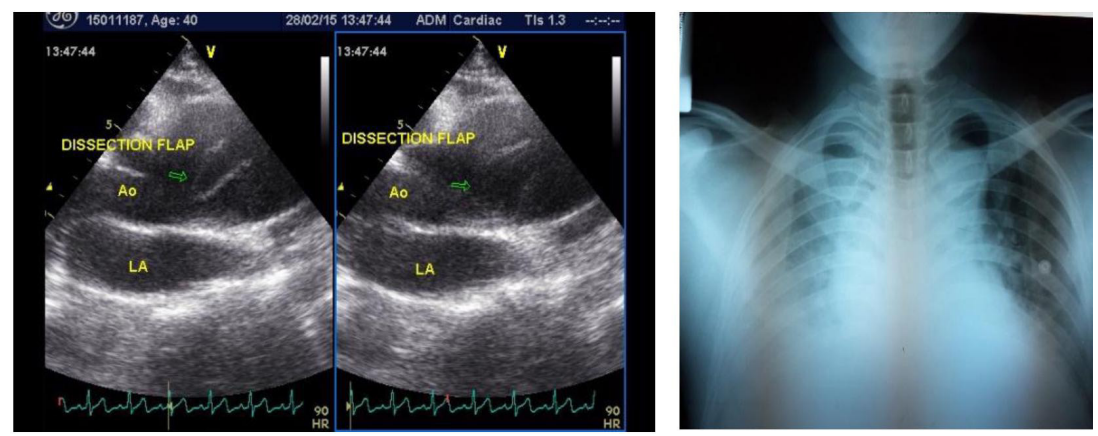

Figure 2 (A) Intimal flap on ascending aorta was shown in transthoracic echocardiography (Standford type A AD), with left ventricle hypertrophy, concentric hypertrophy, dilatation of right atrium and right ventricle, severe aortic regurgitation, moderate mitral regurgitation, severe tricuspid regurgitation, moderate pulmonary regurgitation and moderate pulmonary hypertension. (B) Mediastinum widening, cardiomegaly and congestive pulmonum on plain chest radiograph
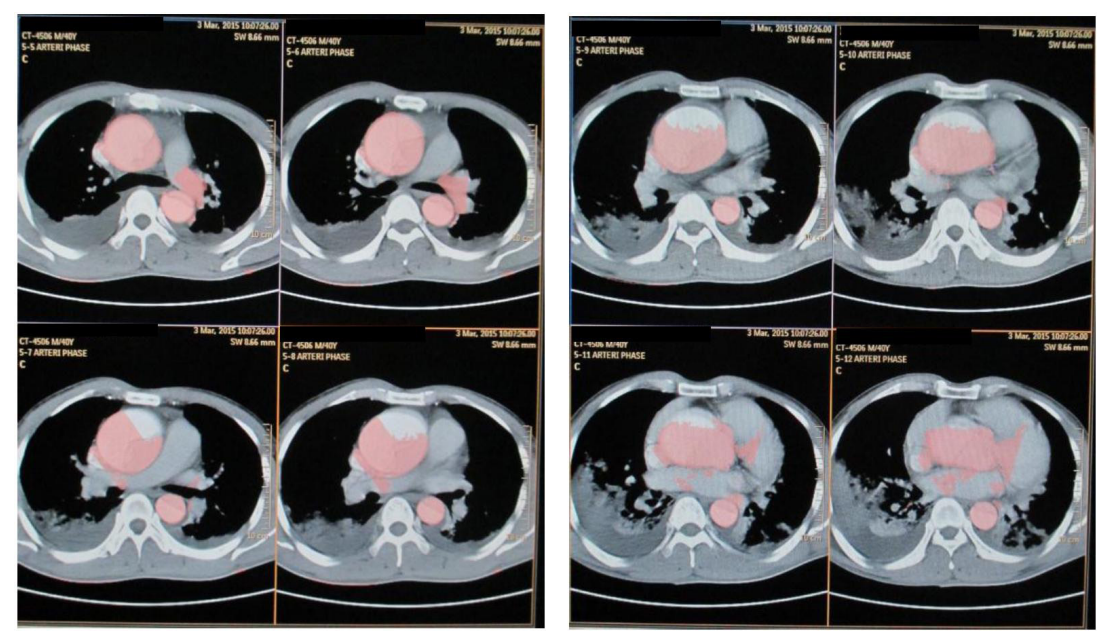

Figure 3 Contrast enhanced-CT scan axial image showed a Stanford type A aortic dissection, dissection flap involving the ascending aorta and extend to descending aorta and abdominal aorta

Initial lab results showed a white blood cell count of 7,160 $\mu \mathrm{L}$, haemoglobin of $10,6 \mathrm{~g} / \mathrm{dL}$, platelets of $172,000 \mu \mathrm{L}$, creatinine of $1.76 \mathrm{mg} / \mathrm{dL}$, AST of
$112.5 \mathrm{U} / \mathrm{L}$, ALT of $38 \mathrm{U} / \mathrm{L}, \mathrm{CKMB}$ of $9.55 \mathrm{IU} / \mathrm{mL}$, Troponin T of $>2000 \mathrm{ng} / \mathrm{mL}$, LDH of $1149 \mathrm{U} / \mathrm{L}$.

An electrocardiogram examination showed a $\mathrm{T}$ inversion in III-AVF (inferior lead) and occasional ventricular extrasystole, which showed inferior ischemia (Figure 1). A thoracic echocardiography was performed after the patient admitted to general hospital, and showed an intimal flap on ascending aorta (Standford type A AD), with left ventricle hypertrophy, concentric hypertrophy, dilatation of right atrium and right ventricle, severe aortic regurgitation, moderate mitral regurgitation, severe tricuspid regurgitation, moderate pulmonary regurgitation and moderate pulmonary hypertension.

The plain chest radiograph showed mediastinum widening, cardiomegaly and congestive pulmonum (Figure 2). Then the computed tomography (CT) angiography showed the presence of a Stanford type A AD (De Bakey type I) (Figure 3 and 4). Consultation and information for emergency surgery have been given to both the patient and her relatives but they refused and decided to take conservative therapy only. The patient survived and received a long term monitoring.

\section{DISCUSSION}

Acute $\mathrm{AD}$ is one of the most common lifethreatening aortic disorder. ${ }^{4}$ Although $\mathrm{AD}$ is uncommon, it frequently has a fatal outcome. Therefore rapid diagnosis and treatment of $\mathrm{AD}$ are very important. ${ }^{4} \mathrm{~A}$ tear in the intimal layer which allows blood entering the aortic wall caused AD, and create an intramural hematoma that progresses distally in the aorta. ${ }^{6}$ A previous study reported that, regarding the anatomic location of the dissection, $60 \%$ of cases affecting the ascending aorta, $10 \%$ in the aortic arch, and $30 \%$ in the descending aorta. ${ }^{7}$ Hypertension (72\%) and atherosclerosis (30\%) are the most frequent risk factors of acute AD. The patient in case presented did not have any known illness nor family history, only with history of cigarettes smoking since years before.

The Stanford and the DeBakey classification system are the most common $\mathrm{AD}$ classification use. Stanford type A affects the ascending aorta ( $\pm 65 \%$ cases), as was in this case presented herein, and type $B$, occurring distal to the subclavian artery and not affecting the ascending aorta. ${ }^{10}$ The DeBakey classification consists of 3 dissections type: Type I affects the ascending aorta, aortic arch, and descending aorta; Type II affects the ascending aorta; Type III affects the descending aorta distal to the left subclavian artery; Type IIIa starts distal to the left subclavian artery and extend proximally and distally above the diaphragm. Type IIIb starts 

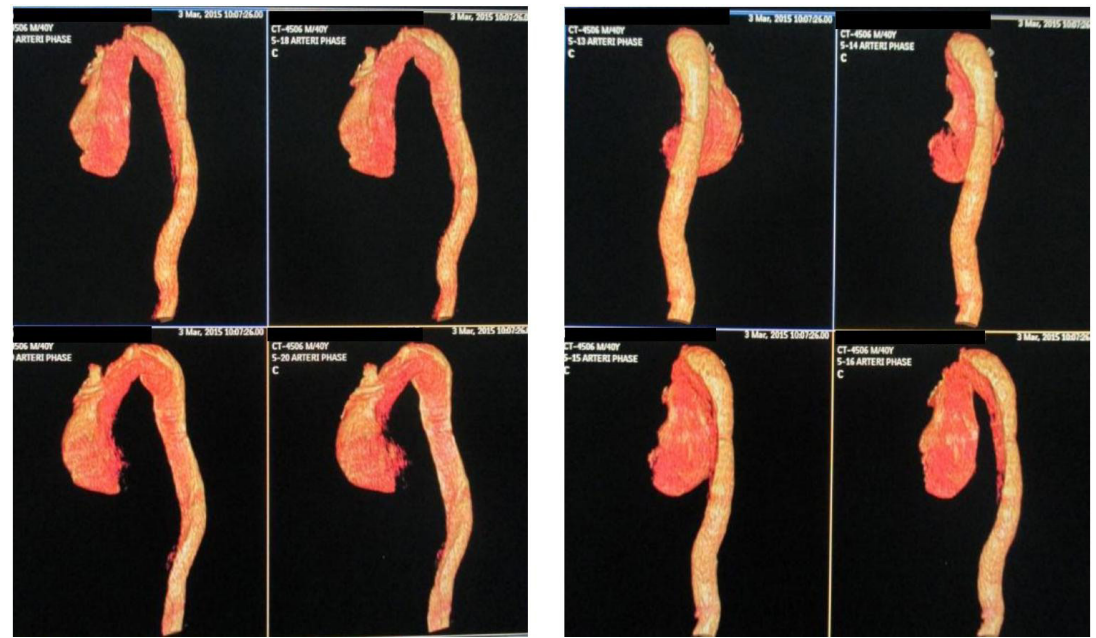

Figure 4 3D reconstruction showed an intimal dissecting flap starts from ascending aorta and extends into the descending thoracic and abdominal aorta

distal to the left subclavian artery and extends only distally sometimes below the diaphragm. ${ }^{6}$

$\mathrm{AD}$ has varying presentation. The symptoms of $\mathrm{AD}$ may be variable and can be similar with other conditions such as myocardial ischemia. ${ }^{4}$ Patients with $\mathrm{AD}$ often present with acute onset chest or back pain, usually defined as sharp or tearing and migrating pain. ${ }^{4}$ Followed by sympathetic activity associated with sweating and also associated with dyspnea and pulmonary oedema. ${ }^{5,8}$ Classic symptoms such as murmur of aortic regurgitation, blood pressure and pulse difference may help early diagnosis of $\mathrm{AD}$, but in mostly these are absent, leading to misdiagnosis. ${ }^{5}$ ECG features of acute ischaemia or acute myocardial infarction were found in about $20 \%$ of patients with type A dissection have. ${ }^{4}$ However, mostly showed non-specific changes in ST-T segment, and only one-third of the patients with $\mathrm{AD}$ have a normal ECG. ${ }^{4}$

In the present case, $\mathrm{AD}$ was not considered at first presentation because of the low index of suspicion. Diagnosis of AMI is based on at minimum two of the following criteria: classical symptoms, changes of ST-T segment in serial ECG, and increased cardiac enzymes. The patient in the case presented matched the criteria. That is why this patient received AMI therapy before. Fortunately, type A AD was confirmed by transthoracic echocardiography and CTA. Some case reports of a Standford type A AD in concomitant with AM. ${ }^{5}$ Whether cases of AD mimicking AMI or case of concomitant incident of both conditions.

These condition might be due to orifice occlusion which obstruct coronary arteries caused by bulging of the dissected false lumen, distal thrombosis, large perfusion through the false channel caused by intimal detachment at the coronary orifice, extension of dissection into the coronary artery, obstruction of the coronary artery orifice during diastole by the flailing intimal flap, and right coronary artery extension of a proximal AD flap. ${ }^{9}$

Widening of mediastinum and signs of cardiac failure due to AD may present on chest X-ray, as in this case. But many studies have shown that chest $\mathrm{X}$-ray does not help distinguish between myocardial infarction and $\mathrm{AD}$. Imaging procedures with CT angiography, magnetic resonance (MR) angiography, and transoesophageal echocardiography (TEE) are validated and sensitive of confirming the diagnosis in over $90 \%$ of cases. ${ }^{6}$

In this case, instead of TEE, transthoracic echocardiography was performed. This noninvasive examination help to detect an ascending AD flap for the AD diagnosis with $59 \%-83 \%$ sensitivity and $63 \%-93 \%$ specificity. ${ }^{2}$ For the diagnosis of a type A dissection transthoracic echocardiography sensitivity is between $78 \%$ and $100 \%$, but only $31 \%-55 \%$ for descending aorta dissections. ${ }^{2}$ In identifying an intimal flap TEE is reported to have a sensitivity of $94 \%-100 \%$ and a specificity of $77 \%-100 .^{2}$ Both can demonstrate all relevant findings-intimal flap, aneurysm, aortic valve involvement, acute aortic insufficiency, LV dilatation, coronary dissection, and pericardial effusion. ${ }^{3}$

Magnetic resonance (MR) angiography and computed tomographic angiography allow precise measurements of aortic diameters. Compared with CT, MR gives a high-resolution multiplanar view, great vessel examination and with no radiation, but due to it's longer acquisition time and difficulty in monitoring, for unstable patients and patients with implanted electronic devices, it cannot be performed. And it cannot visualise calcification of the arterial wall or intimal and the accuracy largely depend on the operator. ${ }^{1,2,3}$

High diagnostic accuracy, short acquisition time and vast availability are the advantages of CT. ${ }^{2}$ In approximately $70 \%$ of cases of $\mathrm{AD}$ the classic intimal flap is seen in CTA. ${ }^{2}$ On CTA, often found an flap in the intima separating true lumen and a false lumen, called the "cobweb sign," and the "beak sign.." If AD affection the entire intima, a circumferential intimal flap occurs which is called a "windsock" appearance, a filiform shaped narrow true lumen with an intimointimal intussusception. A calcified false lumen with mural calcification of the false lumen can be seen in chronic dissections. ${ }^{2}$ If a secondary dissection occurs within one of the channels, three-channel dissection in "MercedesBenz sign" can be seen with the resultant intimal flaps. ${ }^{2}$ As in this case, the diagnosis was confirmed by transthoracic echocardiography and computed tomographic angiography. 
The most important thing is to maintain a high suspicion. If a patient had a hypertension history, presents with acute and severe symptoms or signs suggestive of vascular origin, include chest pain, then $\mathrm{AD}$ should be considered and appropriate investigation and management should be done. ${ }^{6}$ In 2010, based on historical and examination features a guideline for $\mathrm{AD}$ diagnosis was made by the American Heart Association (AHA) and American College of Cardiology (ACC), where patients divided to 3 groups of low risk (score 0 ), intermediate-risk (score 1), and high-risk (score 2 and 3). The sensitivity of this guideline is $95,7 \%$ with no documented specificity. Plasma D-dimer elevation was suggested having a role in the diagnosis of $\mathrm{AD} .{ }^{10}$ The combination of $\mathrm{AD}$ risk score and plasma D-dimer level hoped to be able to facilitate the diagnosis of $\mathrm{AD}$ and in the end improve $\mathrm{AD}$ patients final outcome. ${ }^{4,5,9}$

On type A AD, should be considered to have emergency surgery and not advisable for conservative treatment due to its catastrophic complications. A cardiopulmonary bypass in deep hypothermia at $26^{\circ} \mathrm{C}$ with total circulatory arrest should be performed immediately. The implantation of a stent via the femoral artery may also necessary due to the descending thoracic aorta involvement. ${ }^{6,8}$ Unfortunately patient in the case presented refused to take the emergency surgery as suggested and asked for conservative therapy. The patient survived and received a long term monitoring.

\section{CONCLUSION}

It is still challenging to differentiate AD and another acute chest emergency. Since clinical presentation and laboratory result may be similar between those illnesses. Therefore high index of suspicion with careful history taking, and further imaging examination should be considered to increase the patient outcome.

\section{CONFLICT OF INTEREST}

The authors declare there is no conflict of interest regarding the publication of the case report.

\section{ETHICS IN PUBLICATION}

The patient had received signed inform consent regarding publication of the medical data in journal article.

\section{REFERENCES}

1. Fattori R, Lovato L. Chapter 24: The Thoracic Aorta: Diagnostic Aspects. In: Adam A, Dixon AK, Gillard JH, Schaefer-Prokop CM, eds. Grainger and Allison's Diagnostic Radiology Vol I. 6th ed. China, Churchill Livingstone Elsevier, 2015; 553-590.

2. McMahon MA, Squirell CA. Multidetector CT of AD: A Pictorial Review. RadioGraphics. 2010; 30:445-460. doi: 10.1148/rg.302095104.

3. Claussen CD, Miller S, Riessen R, Fenchel M, Kramer U. Chapter 10: AD. In: Direct Diagnosis In Radiology Cardiac Imaging. Stuttgart, Theme; 2008: 259-262.

4. Hawatmeh A, Arqoub AA, Isbitan A, Fayez S. A case of ascending $\mathrm{AD}$ mimicking acute myocardial infarction and complicated with pericardial tamponade. Cardiovasc Diagn Ther. 2016;6(2):166-171. doi: 10.21037/ cdt.2015.11.06.

5. Zheng Z, Ye Z, Huang Y, Xu J, Cai R, Zhan H. A case report of acute myocardial infarction concomitant with Standford type B aortic dissection. J Car Dis Res. 2013: 251e253 doi: 10.1016/j.jcdr.2013.12.002.

6. Cai J, Cao Y, Yang K, Zhul YS. Inferior myocardial infarction secondary to aortic dissection associated with bicuspid aortic valve. Cardiol Res. 2012;3(2):94-96. doi: 10.4103/0975-3583.95370.

7. Wang JY, Chen H, Song D, Su X Acute Myocardial Infarction Secondary to Aortic Dissection. J Vasc Med Surg. 2016: 255. doi:10.4172/2329-6925.1000255.

8. D’Aloiaa A, Vizzardia E, Bugattia S, Magatellia M, Bonadeia I, Rovettaa R, et al. A Type A Aortic Dissection Mimicking an Acute Myocardial Infarction. Cardiol Res. 2012;3(2):94-96. doi:10.4021/cr151w.

9. Martin JFV, Andrade LG, Loureiro AAC, Fernandes M, Braile DM. Myocardial infarction and aortic dissection: an important differential diagnosis. Braz J Cardiovasc Surg. 2004;19(4):386-390.

10. Danbauchi SS, Ibinaiye PO, Anhiam CA, Alhassan MA. DeBakey type 1 acute aortic dissection presenting as inferior myocardial infarction: A case report and review of literature. Int Sur. 2013;3(2):12-19. doi: 0.4103/2278-9596.122945.

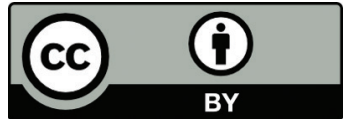

This work is licensed under a Creative Commons Attribution 